\title{
G Research Square \\ Pediatric solid organ transplantation in the era of COVID-19: A follow-up study
}

\section{Mojtaba Shafiekhani}

Department of Clinical Pharmacy, Faculty of Pharmacy, Shiraz University of Medical Sciences, Shiraz, Iran

Kourosh Kazemi

Shiraz Transplant Research Center, Shiraz University of Medical Sciences, Shiraz, Iran

\section{Ali Bahador}

Shiraz Transplant Research Center, Shiraz University of Medical Sciences, Shiraz, Iran

\section{Mohammad Hadi Imanieh}

Department of Pediatrics Gastroenterology and Hepatology, Shiraz University of Medical Sciences, Shiraz, Iran

\section{Parisa Karimzadeh ( $\sim$ karimzadeh.parisa1991@gmail.com )}

Shiraz Transplant Research Center, Shiraz University of Medical Sciences, Shiraz, Iran

\section{Research Article}

Keywords: COVID-19, liver transplantation, kidney transplantation, pediatrics

Posted Date: February 25th, 2021

DOl: https://doi.org/10.21203/rs.3.rs-236136/v1

License: (c) (i) This work is licensed under a Creative Commons Attribution 4.0 International License.

Read Full License 


\section{Abstract}

\section{Background:}

We aimed to evaluate the impact of COVID-19 outbreak on pediatric transplant outcomes and determine whether to continue pediatric transplant activity or not, and how policies intended our center has been effective in preventing COVID-19 among organ transplant recipients.

\section{Methods:}

We conducted a single-center, retrospective, cohort study of hospitalized pediatrics after organ transplantation at Shiraz transplant center since March to August 2020. All liver and kidney transplanted children were included the study and their laboratory and clinical related COVID-19 characteristics were followed up till 3 months after transplantation during hospitalization period and then weekly by the transplant committee.

Results:

Fifty-one patients underwent transplantation including 11 kidney and 40 liver recipients. The mean age of the pediatric cases was $6.72 \pm 5.47$ years. A total of 11 patients died due to post-transplant complications, while none of the patients presented any sign or symptoms in favor of COVID-19 in the hospital course after transplantation. Six transplants including 2 kidney and 4 liver were canceled when positive PCR tests were detected in their donors before the surgery. In the three months of follow up, two patients presented with symptoms including high grade fever, malaise, rhinorrhea, and GI symptoms. Both patients had two negative PCR, and no radiologic or laboratory results regarding COVID-19 were also detected. One had positive influenza PCR, while the second one had a positive serologic test for EBV; $C T$, computed tomography

\section{Conclusion:}

transplant programs could continue their activities during the COVID-19 pandemic with specific case selection, accurate screening methods and following protective protocols.

\section{Introduction}

The global outbreak of the severe acute respiratory coronavirus 2 (SARS-CoV-2) has emerged as a lifethreatening infection, declared as a global pandemic by the Center for World Health Organization (WHO) on March 11(1). According to WHO data, there have been reports of a total of 35,109,317 confirmed cases of COVID-19, including 1,035,341 deaths worldwide, on 5 October 2020 (3:54pm CEST); of them, 471,772 cases and 2,6975 deaths were registered in Iran(2).

Although pediatric data regarding COVID-19 is limited, published studies suggest a less than $5 \%$ involvement of children ( $<18$ years-old) with a less severe manifestations in comparison to adults $(3,4)$. 
However, severe outcomes have been reported in children including hospitalization, ICU admission, and death (5). Moreover, children with underlying conditions such as chronic lung disease (including asthma), cardiovascular disease, and immunosuppression are at a higher risk of severity of the disease (3).

Solid organ transplant recipients, normally maintained in an immunosuppressed state to avoid organ rejection, are expected to be more susceptible for COVID-19 due to their immune status(6). Besides, they might have poorer outcomes in the case of COVID-19 due to their comorbidities, while their immunosuppressive management is also of a great concern $(7,8)$. Therefore, dealing with this situation with respect to issues around donors or recipients, and also approaches regarding new transplant should be aligned with international recommendations.

The aim of the present study was to evaluate the impact of COVID-19 outbreak on pediatric transplant outcomes, whether to continue pediatric transplant activity or not, and transplant recipients diagnosed with COVID-19 among liver and kidney transplanted children who referred to our center as the only tertiary referral hospital in Iran during March 27, 2020 and August 27, 2020 based on the clinical and laboratory data.

\section{Patients And Methods}

\section{1 | Study design}

This retrospective study was done from March to August 2020 in Shiraz transplant center, which is affiliated to Shiraz University of Medical Sciences as a tertiary referral center for pediatric transplantation in Shiraz, Iran. All liver and kidney transplanted patients under 18 years old were included the study and their laboratory and clinical characteristics were followed up till 3 months after their transplant weekly by the transplant committee. This study was approved by the ethics committee of Shiraz University of Medical Sciences and written informed consent was obtained from all the patients' parents or guardians.

\subsection{Data Collection}

Medical and laboratory records from transplantation till 3 months later were extracted from available electronic inpatient medical record databases and reviewed to obtain demographic, clinical, laboratory, and therapeutic information as well as post-transplant complications and outcomes.

\section{3 | Pre transplant evaluation policies:}

In the beginning, all transplant candidates and donors were accurately evaluated by Pediatric Infectious Diseases (ID) specialist regarding COVID-19 symptoms. A normal spiral chest computed tomography (CT) as well as two negative nasopharyngeal swabs for SARS-CoV-2 with 48-hour interval was required from both living donor and recipients before planned hospitalizations or procedures. However, one negative nasopharyngeal swab for SARS-CoV-2 without any suggestive COVID-19 symptoms is enough from cadaver or in case of emergency. A confirmed case of COVID-19 was defined 
by a positive laboratory testing of SARS-CoV-2 by reverse transcription polymerase chain reaction (RTPCR) from a nasopharyngeal sample or highly suggestive symptoms with radiological findings according to Pediatric ID specialist opinion.

\subsection{Specific COVID-19 management (COVID-19 protocol):}

All transplanted patients were visited daily by a transplant surgeon, pediatric gastroenterohepatologist or pediatric nephrologists and a Pediatric ID specialist after transplantation till hospitalization period and routinely evaluated for any clinical signs or symptoms suggestive of COVID19. If an infection was suspected, alongside with SARS-CoV-2 PCR, laboratory tests including complete blood count (CBC), Erythrocyte Sedimentation Rate (ESR), baseline serum C-reactive protein (CRP), lactate dehydrogenase (LDH), ferritin, D-dimer, creatine phosphokinase (CPK), and procalcitonin were sent. In the confirmed case of COVID-19 diseases, management and immunosuppressive changes were done according to published scientific guidelines (9-11).

\section{5 | Follow-ups:}

Outpatient visits were performed both in person in the clinic weekly by the transplant surgeon, pediatric gastroenterohepatologist or pediatric nephrologists, while facial surgical masks were worn by health-care professionals, patients, and parents at all time and telephone pre-triage follow-ups were also done as remote patient monitoring, asking about suggestive symptoms of COVID-19 infection including ongoing fever, gastrointestinal vs. respiratory symptoms in the patient or in the caregiver. This telephone pre-triage was continued until 3 months after their transplantation and extended to the hospital, if needed.

\subsection{Statistical analysis}

Categorical variables were described as frequency rates and percentages, and continuous variables were described using mean, median, and interquartile range (IQR) values. Means for continuous variables were compared using independent group t-tests when the data were normally distributed. All statistical analyses were performed using SPSS (Statistical Package for the Social Sciences) version 20.0 software (SPSS Inc). A p value of $<0.05$ was regarded as statistically significant.

\section{Results}

Fifty-one patients underwent transplantation including 11 kidney and 40 liver recipients during the study period from March 27, 2020 and August 27, 2020.The demographic data are included in Table 1. As shown, the mean age of the pediatric cases was $6.72 \pm 5.47$ years (range, 12 to 18 years). In total, $33(64.7 \%)$ patients were female in comparison to $18(35.3 \%)$ male patients. 
Regarding the underlying disease presented in Table1, among liver transplant patients, Biliary atresia accounted for the majority of cases, followed by Progressive familial intrahepatic cholestasis (PFIC), Wilson, while Focal segmental glomerulosclerosis (FSGS) was the main cause of kidney transplant among our patients. Among liver transplant recipients, the median Pediatric end-stage liver disease (PELD) score was $21.20 \pm 8.8$ (6-49). The baseline laboratory results are detailed in Supplementary Table1. Twenty-four transplantations (47.1\%) were from living-donor and $27(52.9 \%)$ were from cadaverdonor.

At the end of the study, 40 (78.4\%) patients were discharged with median ICU admission length of 27.97 days (13-77 days). Eleven patients died after liver transplant due to post-transplant complications including bleeding (1), portal vein thrombosis (PVT) (1), Disseminated intravascular coagulation (DIC) (4), and graft failure (5), whereas all kidney transplant patients were alive.

None of the patients presented any sign or symptoms in favor of COVID-19 in the length of hospital stay. Six transplants including 2 kidney and 4 liver were canceled when positive PCR tests were detected in their donors before the surgery.

Among these patients, $41(80 \%)$ were not readmitted after discharge from the hospital in the three months of our follow up. Among other 10 patients, two were admitted due to ascites, four due to elevated liver enzyme and creatinine level, and four due to bacterial infections.

In the three months of follow up, two patients presented with symptoms including high grade fever, malaise, rhinorrhea, and GI symptoms and were evaluated with suspicion to COVID-19 by pediatric ID specialist. Both patients had two negative PCR, and no radiologic or laboratory results such as lymphopenia or decrease in 02 saturation level were detected. One of them had positive influenza PCR test and were treated with Oseltamivir, while the second one had a positive serologic test for EBV which was treated by Acetaminophen and reduction in immunosuppressive regimen.

\section{Discussion}

As immunosuppression and comorbidities predominantly afflict the solid organ transplant recipients, especially pediatric groups, COVID-19 is a matter of concern for many pediatric transplantation centers. At the moment, little is known about the exact impact of COVID-19 in solid transplant patient outcome. In our study, we retrospectively reviewed 51 pediatric transplanted patients after their surgery with a three month follow up. Our findings showed a slight reduction (26\%) in the rate of transplantation in our center compared to last year as well as no COVID-19 involvement in the new organ recipients.

From the pediatric perspective, it could be assumed that children have a less severe disease in comparison to adults, and they corresponded with only $5 \%$ of the total number of patients involved with COVID-19 in China (12). While this could not be related to transmission chance since viral transmission in children is mentioned to be apparently similar to adult group in an epidemiological analysis by Qifang et al. of 391 cases and their 1286 close contacts (12). 
Some hypotheses may explain the benign course of COVID-19 in pediatric cases compared with adults. First, viral clearance is more rapid in children which may lead to inflammatory response reduction which appears to be particularly important drivers of tissue damage during infection $(13,14)$. Second, children do not carry degenerative features of aging that is considered as COVID-19 risk factor(13,14). Milder pattern of the disease may also be related to the lower maturity and the binding capacity of the angiotensin converting enzyme II, probably the virus cell receptor (13).

Immunocompromised patients' reports are also few; this could illustrate their lower chance of involvement with the virus. Although earliest report of liver transplant children was available in Bergamo (15), their negligible symptoms suggested that the immunological impairment itself may be the cause of blunting the inflammatory cascades and cytokine release since the inflammatory reaction enhances the damage of the disease(6). Another case-control study by Chaudhry and his colleague on the clinical characteristics and outcomes of COVID-19 in solid organ transplant recipients associated the increasing age and clinical severity with mortality. While transplant status itself was not considered as a contributing factor with mortality(6). Another study demonstrated that Tacrolimus, widely used in transplant patients, strongly inhibited the growth of human coronavirus SARS-CoV, too (16). Furthermore, tight social distancing which is usually experienced by immunocompromised patients is considered as the main factor in their lower infection risks (17). Our preliminary experience, in agreement with recent data from of China and Italy which showed that none of the patients was followed for transplantation or autoimmune liver disease, developed a clinical pulmonary disease, except for some positive tests for SARSCoV-2. They assert that immunosuppressed patients are not at a higher risk of severe complications in comparison to the general population, both in children and adults (18).

Pediatric transplantation (0-17 years old) is a highly complex procedure that makes coordination of resources and specialized professionals of a great importance (19). A total rate of 108 pediatric transplant per year reported an activity of approximately 317 pediatric transplants from 2017 to 2019, while a $29.2 \%$ reduction was seen in this period in our center, which is compatible with the result of European centers that show COVID-19 pandemic substantial negative effect on pediatric transplantation activity as well as outpatient visits due to the fear of SARS-CoV-2 transmission risk, and shortage of hospital bed capacity and staff. On the other hand, it is noteworthy to mention the liver transplantation center of Hong Kong which shifted its resources to SARS patients during the SARS outbreak and performed no transplants for 6 months (20). This approach directly affected the quality of care in transplanted patients and liver transplant candidates though no case of severe pneumonia was also recorded (20). The long-term continuation of these limitations surely has dire consequences on pediatric transplant recipients as well as children on the transplant waiting list, reducing their access to close monitoring and follow ups. Therefore, optimizing the resource in specialized transplant center and establishing guidelines in terms of pediatric transplant recipients and candidates during the COVID-19 pandemic are required. D'antiga also suggested that coronaviruses have not been shown to cause a more severe disease in immunosuppressed patients, so that there is no reason to postpone lifesaving treatments, such as transplantation, both in children and in adults during coronavirus outbreaks (15). 
However, as the only pediatric transplant center in the country, our centers had to remain active during the period and the living donor was reserved for urgent cases, while cadaveric donors were available with specific adaptations of the protocols for COVID-19 screening, although in both cases the accessibility was notably reduced. For complex patients, prioritization of which patients in the list for transplant in the period is regarded to Higher PELD score and acute liver failures. Besides, outpatient activities are continued both in person, and through telemedicine. In our protocol, a normal spiral chest CT as well as two negative nasopharyngeal swabs for SARS-CoV-2 with 48-hour interval were taken from both living donors and recipients before planned hospitalizations or procedures which exclude 6 donors from the list due to their positive PCR test.

There is no final opinion regarding pre-transplant evaluation. Some centers use PCR and some Antibody test, while others only rely on spiral chest $C T(21,22)$. The timing of tests varies from one week to one day before transplant in different studies(23). In our study, the donor was excluded from transplant list if he/she presented with a positive PCR test, while other centers checked if PCR became negative or Antibody was presented (24).

This study has limitations. Since we are the only pediatric transplant center in Iran, our results cannot be compared with other less equipped centers to generalize. Even though our median follow-up was three months, long-term follow up needs further studies.

\section{Conclusion}

In conclusion, despite the greater proportion of coexisting conditions and immune suppression, solid organ transplant recipients seem not to have greater risk of COVID-19 involvement. Long term evaluation as well as multi-center data is also needed for further determination. Therefore, transplant programs could continue their activities during the COVID-19 pandemic with specific case selection, accurate screening methods while following protective protocols.

\section{Declarations}

\section{Ethics approval and consent to participate:}

The present study was approved by the Medical Ethics Committee of Shiraz University of Medical Sciences. The purpose of this study was completely explained to the patient and their parents and were assured that their information will be kept confidential by the researchers. The written Informed consent was obtained from the patient's parents.

\section{Consent for publication}

The written Informed consent was obtained from the patient's parents.

\section{Availability of data and material}


The datasets generated and/or analysed during the current study are not publicly available due their containing information that could compromise the privacy of research participants but are available from the corresponding author on reasonable request.

\section{Competing interests}

The authors declare that they have no competing interests.

\section{Funding}

This study was financially supported by the Vice-Chancellery of Research and Technology of Shiraz University of Medical Sciences.

\section{Authors' contributions}

MS and KK designed the study. PK collected the data carried out the statistical analysis. RS drafted the manuscript. MS and AB revised and proofread the manuscript. All authors read and approved the final version of the manuscript.

Acknowledgement: The authors would like to thank Shiraz University of Medical Sciences, Shiraz, Iran and also Center for Development of Clinical Research of Namazi Hospital and Dr. Nasrin Shokrpour for editorial assistance.

\section{Conflict of Interest:}

The authors of this manuscript have no conflict of interest to disclose as described by Pediatric Transplantation Journal.

\section{References}

1. Sohrabi C, Alsafi Z, O'Neill N, Khan M, Kerwan A, Al-Jabir A, et al. World Health Organization declares global emergency: A review of the 2019 novel coronavirus (COVID-19). International Journal of Surgery. 2020.

2. updates. WHOCdC-oL. [

3. Covid C, COVID C, COVID C, Bialek S, Gierke R, Hughes M, et al. Coronavirus Disease 2019 in Children -United States, February 12-April 2, 2020. Morbidity and Mortality Weekly Report. 2020;69(14):422.

4. Kelvin AA, Halperin S. COVID-19 in children: the link in the transmission chain. The Lancet Infectious Diseases. 2020.

5. Cai J, Xu J, Lin D, Xu L, Qu Z, Zhang Y, et al. A Case Series of children with 2019 novel coronavirus infection: clinical and epidemiological features. Clinical Infectious Diseases. 2020.

6. Chaudhry ZS, Williams JD, Vahia A, Fadel R, Parraga Acosta T, Prashar R, et al. Clinical characteristics and outcomes of COVID-19 in solid organ transplant recipients: A cohort study. 
American Journal of Transplantation. 2020.

7. Mirjalili M, Shafiekhani M, Vazin A. Coronavirus disease 2019 (COVID-19) and transplantation: pharmacotherapeutic management of immunosuppression regimen. Therapeutics and Clinical Risk Management. 2020;16:617.

8. Zhong Z, Zhang Q, Xia H, Wang A, Liang W, Zhou W, et al. Clinical characteristics and immunosuppressant management of coronavirus disease 2019 in solid organ transplant recipients. American Journal of Transplantation. 2020.

9. Teoh CW, Gaudreault-Tremblay M-M, Blydt-Hansen TD, Goldberg A, Arora S, Feber J, et al. Management of Pediatric Kidney Transplant Patients During the COVID-19 Pandemic: Guidance From the Canadian Society of Transplantation Pediatric Group. Canadian Journal of Kidney Health and Disease. 2020;7:2054358120967845.

10. Fix OK, Hameed B, Fontana RJ, Kwok RM, McGuire BM, Mulligan DC, et al. Clinical best practice advice for hepatology and liver transplant providers during the COVID-19 pandemic: AASLD expert panel consensus statement. Hepatology. 2020.

11. Foust AM, McAdam AJ, Chu WC, Garcia-Pena P, Phillips GS, Plut D, et al. Practical Guide for Pediatric Pulmonologists on Imaging Management of Pediatric Patients with COVID-19. Pediatric Pulmonology. 2020.

12. Bi Q, Wu Y, Mei S, Ye C, Zou X, Zhang Z, et al. Epidemiology and transmission of COVID-19 in 391 cases and 1286 of their close contacts in Shenzhen, China: a retrospective cohort study. The Lancet Infectious Diseases. 2020.

13. Dong Y, Mo X, Hu Y, Qi X, Jiang F, Jiang Z, et al. Epidemiological characteristics of 2143 pediatric patients with 2019 coronavirus disease in China. Pediatrics. 2020.

14. Wu Z, McGoogan JM. Characteristics of and important lessons from the coronavirus disease 2019 (COVID-19) outbreak in China: summary of a report of 72314 cases from the Chinese Center for Disease Control and Prevention. Jama. 2020;323(13):1239-42.

15. D'Antiga L. Coronaviruses and immunosuppressed patients: the facts during the third epidemic. Liver Transplantation. 2020.

16. Carbajo-Lozoya J, Müller MA, Kallies S, Thiel V, Drosten C, Von Brunn A. Replication of human coronaviruses SARS-CoV, HCoV-NL63 and HCoV-229E is inhibited by the drug FK506. Virus research. 2012;165(1):112-7.

17. Yang C, Li C, Wang S. Clinical strategies for treating pediatric cancer during the outbreak of 2019 novel coronavirus infection. Pediatric blood \& cancer. 2020;67(5):e28248.

18. Morand A, Roquelaure B, Colson P, Amrane S, Bosdure E, Raoult D, et al. Child with liver transplant recovers from COVID-19 infection. A case report. Archives de Pédiatrie. 2020.

19. Jara P, Baker A, Baumann U, Borobia A, Branchereu S, Candusso M, et al. Cross-cutting view of current challenges in paediatric solid organ and haematopoietic stem cell transplantation in Europe: the European Reference Network TransplantChild. Orphanet journal of rare diseases. 2020;15(1):1-9. 
20. Chui A, Rao A, Chan H, Hui A, editors. Impact of severe acute respiratory syndrome on liver transplantation service. Transplantation proceedings; 2004: Elsevier.

21. Tschopp J, L'Huillier A, Mombelli M, Mueller NJ, Khanna N, Garzoni C, et al. First experience of SARSCoV-2 infections in solid organ transplant recipients in the Swiss Transplant Cohort Study. American Journal of Transplantation. 2020.

22. Pereira MR, Mohan S, Cohen DJ, Husain SA, Dube GK, Ratner LE, et al. COVID-19 in solid organ transplant recipients: Initial report from the US epicenter. American Journal of Transplantation. 2020.

23. Ju C-R, Lian Q-Y, Zhang J-H, Qiu T, Cai Z-T, Jiang W-Y, et al. Recommended prophylactic and management strategies for severe acute respiratory syndrome coronavirus 2 infection in transplant recipients. Chronic Diseases and Translational Medicine. 2020.

24. Boyarsky BJ, Ruck JM, Chiang TPY, Werbel WA, Strauss AT, Getsin SN, et al. Evolving Impact of COVID-19 on Transplant Center Practices and Policies in the United States. Clinical transplantation. 2020:e14086.

\section{Tables}

\section{Table 1. Demographic data of pediatric cases who had undergone liver and kidney transplantation $(\mathrm{N}=51)$}




\begin{tabular}{l|c}
\hline Variable & N or Mean \pm SD \\
\hline Age (years) & $6.72 \pm 5.47$ \\
\hline Sex & $33(64.7)$ \\
\hline Male & $18(35.3)$ \\
\hline Female & $8.79 \pm 5.57$ \\
\hline White blood cells & $0.64 \pm 1.04$ \\
\hline Creatinine $(m g / d L)$ & $161.58 \pm 132.52$ \\
\hline Aspartate transaminase (U/L) & $98.75 \pm 80.93$ \\
\hline Alanine aminotransferase (U/L) & $17.96 \pm 16.88$ \\
\hline Total Bilirubin (mg/dL) & $21.20 \pm 8.88$ \\
\hline PELD score & \\
\hline Transplant type & \\
\hline Liver & $11(21.6)$ \\
\hline Kidney & \\
\hline
\end{tabular}

Patients outcome

\begin{tabular}{|c|c|}
\hline Alive & $40(78.4)$ \\
\hline Expired & $11(21.6)$ \\
\hline \multicolumn{2}{|l|}{ Immunosuppressive regimen } \\
\hline Tacrolimus +prednisolone & $32(62.74 \%)(32.3 \%)$ \\
\hline mycophenolate mofetil +tacrolimus & $9(17.64 \%)$ \\
\hline Cyclosporine +prednisolone & $10(19.60 \%)$ \\
\hline \multicolumn{2}{|l|}{ Induction regimen } \\
\hline Methylprednisolone & $41(80.39 \%)$ \\
\hline Thymoglobulin & $10(19.6 \%)$ \\
\hline \multicolumn{2}{|l|}{ Post-transplant laboratory value) } \\
\hline $\mathrm{O}_{2}$ saturation (\%) & 91 \\
\hline Lymphocytopenia & $5(10.6 \%)$ \\
\hline Lymphocytosis & $13(27.7 \%)$ \\
\hline Elevated CRP & $19(51.4 \%)$ \\
\hline Elevated ESR & $8(22.9 \%)$ \\
\hline Prograf level(ng/dl) & $4.91 \pm 2.21$ \\
\hline Cyclosporine level & $190.34 \pm 34.12$ \\
\hline CMV PCR positive & $4(14.8 \%)$ \\
\hline
\end{tabular}


PELD: Pediatric end-stage liver disease, CRP: C-reactive protein, ESR: erythrocyte sedimentation rate CMV: cytomegalovirus, PCR, polymerase chain reaction 